\title{
Influence of Cationic Polyelectrolytes on the Reaction of 5,5'-Dithiobis(2-nitrobenzoate) with Cyanide Ion
}

\author{
Toshio Ueda and Susumu Harada \\ Research Laboratory for Chemical Fibers, Nitto Spinning Co., Ltd., \\ Koriyama, Fukushima, Japan. \\ Norio IsE \\ Department of Polymer Chemistry, Kyoto University, \\ Kyoto, Japan. \\ (Received December 3, 1973)

\begin{abstract}
KEY WORDS 5,5'-Dithiobis(2-nitrobenzoate) / Cyanide Ion / Polyelectrolyte Acceleration / Diallylammonium Chloride-Sulfur Dioxide Copolymers /
\end{abstract}

It is now well-established that interionic reactions can be greatly enhanced or decelerated by addition of polyelectrolytes and micelles. ${ }^{1}$ It has been pointed out that the electrostatic and, sometimes, the hydrophobic interactions between reactants and polyelectrolytes stabilize the critical complex for reactions between likecharged ionic species so that acceleration can be observed. ${ }^{2}$ We recently reported that the rate enhancement for an $S_{N} A r$ reaction of 3,5-

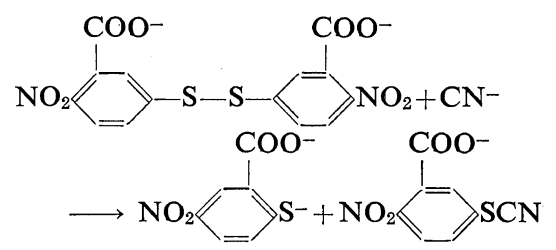

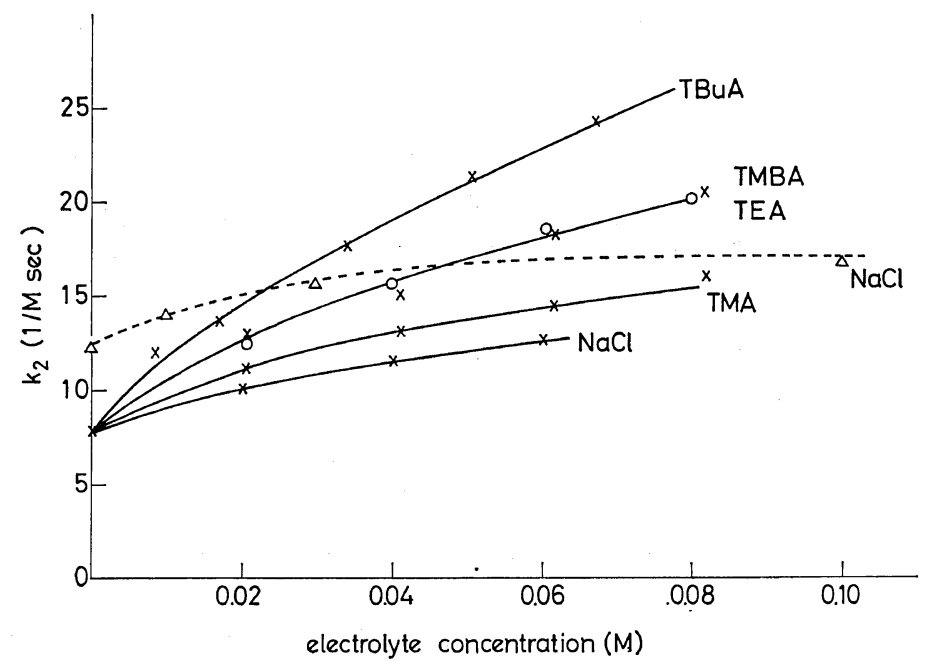

Figure 1. Influence of low-molecular-weight electrolytes on the reaction of 5,5'-dithiobis(2-nitrobenzoate) with $\mathrm{CN}^{-}$: $\mathrm{TBuA}$, tetrabutylammonium chloride; TMBA $(x)$, trimethylbenzylammonium chloride; TEA (O), tetraethylammonium chloride, TMA; tetramethylammonium chloride; [KCN], $5 \times 10^{-4} M$; [DTNB], $6 \times 10^{-5} M$; pH, 10.6; solid curves, $25^{\circ}$; broken curve, $30^{\circ}$. 


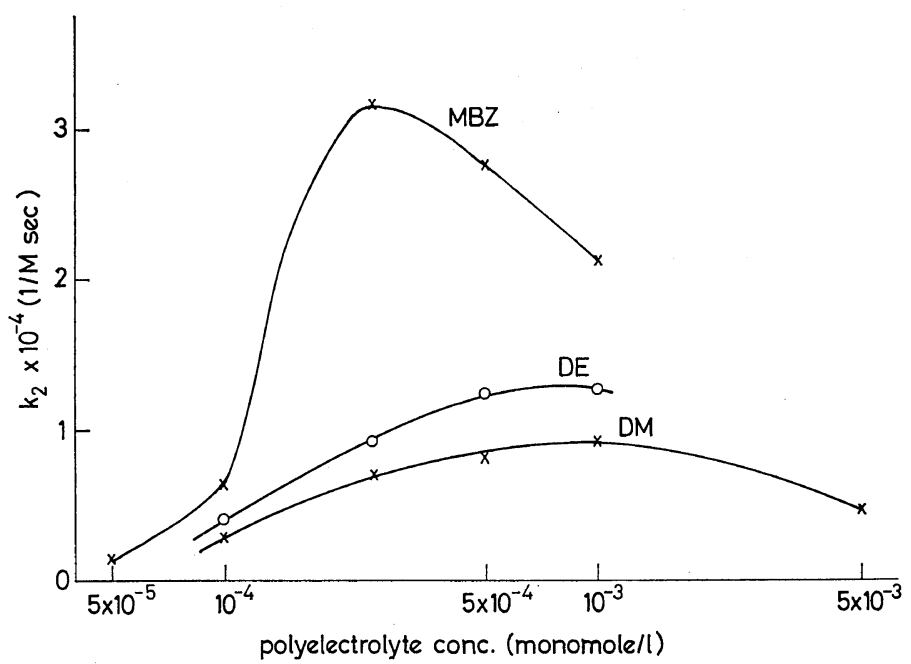

Figure 2. Influence of cationic polyelectrolytes on the reaction of 5,5'-dithiobis(2-nitrobenzoate) with $\mathrm{CN}^{-}$: [DTNB], $6 \times 10^{-5} \mathrm{M} ;[\mathrm{KCN}], 5 \times 10^{-4} \mathrm{M} ; \mathrm{pH}, 10.6 ; 30^{\circ} \mathrm{C}$.

dinitro-4-chlorobenzoate with $\mathrm{OH}^{-}$became larger with increasing hydrophobicity of the cationic polyelectrolytes added. ${ }^{6}$ In this note, we report the study of the well-known reaction of $5,5^{\prime}$ dithiobis(2-nitrobenzoate) [DTNB] with $\mathrm{CN}^{-}$in the presence of cationic polyelectrolytes with various degrees of hydrophobicities. DTNB was synthesized by Ellman ${ }^{7}$ for thiol determination, and reacts easily with strong nucleophiles such as mercapto anions. DTNB reacts slowly with $\mathrm{CN}^{-}$and even more slowly with $\mathrm{OH}^{-}$.

The kinetic measurement were carried out in buffer solutions containing $4.5 \times 10^{-3}-M$ sodium carbonate and $5 \times 10^{-4}-M$ sodium borate. The $\mathrm{pH}$ value was 10.6. The production of 2-nitro-5mercaptobenzoate was followed at $412 \mathrm{~m} \mu$, using a Hitachi RSP-2 stopped-flow apparatus.

Figure 1 gives the second-order rate constant of the reaction in the presence and absence of low-molecular-weight electrolytes. The addition of simple salts enhanced the reaction; this is basically the primary salt effect.

Copolymers of sulfur dioxide and dimethyldiallylammonium chloride (DM), diethyldiallylammonium chloride (DE), or methylbenzyldiallylammonium chloride (MBZ) were added in the reaction system. The methods of preparation of these copolymers were described earlier. ${ }^{8,9}$ The influence of the copolymers on the rate constant is given in Figure 2. The more hydrophobic the copolymers, the larger the rate enhancement: MBZ is most effective and DM shows the smallest acceleration. At an MBZ concentration of $2.5 \times 10^{-4} \mathrm{monomol} / \mathrm{l}$, the acceleration amounted to $2.5 \times 10^{3}$ times. It is obvious that the polycations affect the DTNB anions through both electrostatic and hydrophobic interactions and the $\mathrm{CN}^{-}$through electrostatic interactions. The activated complex is more strongly stabilized through the cooperative action of the electrostatic and hydrophobic interactions than the reactants. According to Brønsted, ${ }^{10}$ this stabilization results in acceleration.

\section{REFERENCES}

1. For a convenient review of this topic, see a review article presented by $\mathrm{N}$. Ise at the Symposium on Polyelectrolytes, Pasadena, May 1973, to be published. Earlier work should be referred to in this review.

2. Hydrophobic interactions have been demonstrated to be important in determining the acceleration for various reaction systems.3,4 However, because of their short-range nature, the hydrophobic interactions do not always give rise to rate enhancement. See, for example, the alkaline fading reaction of phenol- 
T. UEDA, S. HARADA, and N. IsE

phthalein in the presence of polyelectrolytes containing hydrophobic moiety. 5

3. I. Sakurada, Pure Appl. Chem., 16, 263 (1968).

4. H. C. Kiefer, W. I. Congdon, I. S. Scarpa, and I. M. Klotz, Proc. Nat. Acad. Sci., U.S.A., 69, 2155 (1972).

5. T. Okubo and N. Ise, J. Amer. Chem. Soc., 95, 2293 (1973).

6. T. Ueda, S. Harada, and N. Ise, Polymer J.,
6, 319 (1974).

7. G. Ellman, Arch. Biochem. Biophys., 82, 70 (1959).

8. S. Harada and K. Arai, Makromol. Chem., 107, 64 and 78 (1967).

9. T. Ueda, S. Harada, and N. Ise, Polymer J., 3, 476 (1972).

10. J. N. Brønsted and R. Livingston, J. Amer. Chem. Soc., 49, 435 (1927). 